\title{
Moluscos límnicos da microrregião de Belo Horizonte, MG, com ênfase nos vetores de parasitoses
}

\author{
Limnic snails in the microregion of Belo Horizonte, Minas Gerais \\ State, Brazil:a survey emphasizing on vectors of parasitosis
}

\author{
Cecília Pereira de Souza, Laís Clark Lima, Liana Konovaloff Jannotti-Passos, \\ Sueleny Silva Ferreira, Carlos Tito Guimarães, laci Belo de \\ Figueiredo Vieira e Rafael Mariani Junior
}

\begin{abstract}
Resumo Um levantamento malacológico em coleções hídricas de 13 municípios da microrregião de Belo Horizonte, MG, Brasil, foi efetuado para detectar focos de transmissão de esquistossomose e outras parasitoses. De 1990 a 1996 foram coletados 22.066 moluscos dos quais 378 (1,7\%) estavam infectados com trematódeos: Biomphalaria glabrata (7.920), com Schistosoma mansoni (1,9\%), com Echinostomatidae (1,2\%), com Strigeidae (0,6\%), com Cercaria minense $(0,1 \%)$ e Derogenidae (-0,1\%); B. straminea (4.093), com Strigeidae (0,6\%), com Echinostomatidae (0,2\%), com Clinostomatidae $(-0,1 \%)$ e duas cercárias desconhecidas; B. tenagophila (1.338), com Strigeidae (0,1\%); Physa marmorata (1.776), com Echinostomatidae (1,6\%). Os moluscos Biomphalaria peregrina, B. occidentalis, B. schrammi, Drepanotrema depressissimum, D. lucidum, D. cimex, Physa cubensis, Lymnaea columella, Melania tuberculata, Idiopyrgus souleyetianus, Pomacea $\mathrm{sp}$, Anodontites sp e Ancylidae não estavam infectados. Moluscos de 9 municípios estavam infectados com S. mansoni e de 11 com outros trematódeos.
\end{abstract}

Palavras-chaves: Moluscos límnicos. Vetores. Parasitas. Microrregião. Belo Horizonte.

\begin{abstract}
A malacological survey to detect foci of transmission of schistosomiasis and other parasitic diseases was undertaken into water-courses from 13 municipalities of microregion of Belo Horizonte, MG, Brazil. From 1990 to 1996, 22,066 snails were collected. From those, $378(1.7 \%)$ were found infected by trematodes: Biomphalaria glabrata $(7,920)$, infected by Schistosoma mansoni (1.9\%), Echinostomatidae (1.2\%), Strigeidae (0.6\%), Cercaria minense (0.1\%) and Derogenidae $(-0.1 \%) ;$ B. straminea (4,093) infected by Strigeidae (0.6\%), Echinostomatidae (0.2\%), Clinostomatidae $(-0.1 \%)$ and two unidentified cercariae; B. tenagophila $(1,338)$, infected by Strigeidae $(0.1 \%)$ and Physa marmorata $(1,776)$ by Echinostomatidae $(1.6 \%)$. The snails Biomphalaria peregrina, B. occidentalis, B. schrammi, Drepanotrema depressissimum, D. lucidum, D. cimex, Physa cubensis, Lymnaea columella, Melania tuberculata, Idiopyrgus souleyetianus, Pomacea $\mathrm{sp}$, Anodontites sp and Ancylidae were found noninfected. Snails from 9 municipalities were infected by S. mansoni and from 11 by other trematodes.
\end{abstract}

Key-words: Limnic snails. Hosts. Parasites. Microregion. Belo Horizonte.

\footnotetext{
Laboratório de Malacologia, Centro de Pesquisas René Rachou, Fundação Oswaldo Cruz, Ministério da Saúde, Belo Horizonte, MG, Brasil.

Endereço para correspondência: $\mathrm{Dr}^{\mathrm{a}}$ Cecília Pereira de Souza, Centro de Pesquisas René Rachou/FIOCRUZ, Av. Augusto de Lima, 1715, 30190-002 Belo Horizonte, MG, Brasil,

Fax (031) 2953115.

Recebido para publicação em 17/12/97.
} 
No município de Belo Horizonte, região endêmica de esquistossomose, desde o primeiro relato de ocorrência dessa parasitose 24 tem sido efetuados levantamentos malacológicos para a localização e controle dos focos de transmissão5 9101422 .

Nos últimos anos os criadouros de moluscos foram reduzidos devido ao progresso e medidas de urbanização e atualmente os focos, com raras exceções, se encontram na periferia ou municípios vizinhos 5672021 22. Eventualmente, em um criadouro ocorre a substituição de uma espécie hospedeira por outra23. Além dos focos de transmissão de esquistossomose, alguns autores tem registrado ainda as taxas de infecção dos moluscos de água doce com larvas de outros trematódeos não só na microrregião de Belo Horizonte como em outras regiões do Estado1 215161921.

O encontro ao longo de vários anos de larvas de trematódeos, parasitas não humanos, em moluscos de vários municípios da microrregião de Belo Horizonte, demonstra que os ciclos biológicos desses helmintos vem sendo mantidos na região e mostra a necessidade de mais pesquisas para detectar os hospedeiros vertebrados e identificar os vermes adultos.

No presente estudo foram pesquisadas coleções hídricas de 13 municípios da microrregião de Belo Horizonte com os objetivos de detectar a fauna malacológica, principalmente a planorbídica, os focos de transmissão de esquistossomose e detectar quais os gêneros ou espécies de moluscos que atuam como hospedeiros de larvas de trematódeos parasitas não humanos, nessa região. Esse dados são ainda uma contribuição para a elaboração da carta planorbídica do Estado de Minas Gerais.

\section{MATERIAL E MÉTODOS}

As coletas de moluscos foram efetuadas no período de 1990 a 1996 em 13 municípios da microrregião de Belo Horizonte: Belo Horizonte, Pedro Leopoldo, Lagoa Santa, Santa Luzia, Vespasiano, Ribeirão das Neves, Contagem, Sabará, Caeté, Raposos, Nova Lima, Betim e Rio Acima (Figura 1).

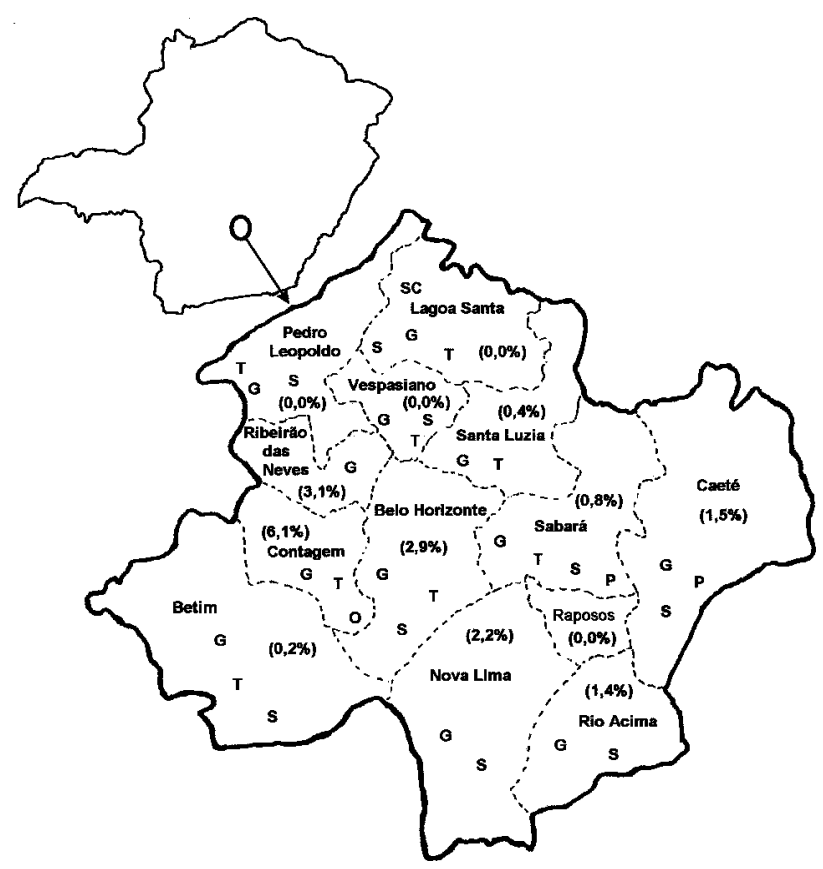

Figura 1 - Distribuição de moluscos do gênero Biomphalaria por município, na microrregião de Belo Horizonte, MG e taxas de infecção de B. glabrata com Schistosoma mansoni (\%): G: B. glabrata; O: B. occidentalis; P: B. peregrina; S: B. straminea; SC: B. schrammi; T: B. tenagophila. 
Os moluscos foram coletados com auxílio de concha de metal, perfurada, de $17 \mathrm{~cm}$ de diâmetro de abertura, $12 \mathrm{~cm}$ de fundo e $8 \mathrm{~cm}$ de altura, com cabo de madeira com cerca de $120 \mathrm{~cm}$ de comprimento. A concha era introduzida 10 vezes em cada criadouro, procedendo-se a lavagem e separação do material coletado.

As coleções hídricas pesquisadas foram escolhidas levando-se em consideração a proximidade das sedes de fazendas, vilas ou povoados e a periferia de cidades.

Foram efetuadas coletas em 14 a 53 coleções hídricas por município, com exceção de Raposos, onde foram pesquisadas apenas 7 coleções devido à dificuldade de acesso a alguns pontos por causa do relevo.

Após a coleta, os moluscos eram lavados e colocados em pequenos sacos de plástico incolor, a seco e levados para o laboratório junto com a ficha de captura, contendo data, local de coleta e tipo de criadouro. No laboratório os moluscos eram contados, medidos e cerca de quatro exemplares anestesiados e fixados com RailletHenry para identificação específica através da morfologia interna 4 13. O restante era examinado individualmente em estereomicroscópio após exposição à luz e posteriormente procedia-se ao esmagamento entre lâminas de vidro, para detectar a presença ou não de larvas de trematódeos. Algumas cercárias foram fixadas com formol a $10 \%$ e mantidas para identificação posterior.

\section{RESULTADOS}

Em 7 anos foram coletados 22.066 moluscos em 308 coleções hídricas de 13 municípios da microrregião de Belo Horizonte, dentre os quais $13.526(61,3 \%)$ pertenciam ao gênero Biomphalaria (Figura 1).O número de moluscos coletados por município, a espécie, gênero ou família são mostradas nas Tabelas 1 e 2. A Tabela 3 apresenta o total de molusco infectado de cada espécie, os trematódeos encontrados e a sistemática dos helmintos, até o momento.

Foram encontradas larvas de trematódeos, parasitas não humanos, em moluscos de 11 municípios: Pedro Leopoldo, 5 espécies; Lagoa Santa, 2; Santa Luzia, 1; Vespasiano, 1; Ribeirão das Neves, 4; Belo Horizonte, 3; Sabará, 2; Caeté, 1; Nova Lima, 1; Betim, 1; Rio Acima, 1.

Dentre o total de moluscos coletados, 7.920 $(35,9 \%)$ eram B. glabrata; $4.093(18,5 \%)$ B. straminea; $1.338(6,1 \%)$ B. tenagophila; 148
$(0,7 \%)$ B. peregrina, $25(0,1 \%)$ B. occidentalis e $2(-0,1 \%)$ B. schrammi (Tabelas 1 e 2$)$

Biomphalaria glabrata foi encontrada em 12 municípios e dentre 7.920 exemplares 156 $(1,9 \%)$ estavam infectados com $S$. mansoni e $154(1,9 \%)$ com larvas de outros trematódeos. (Tabela 3)

As taxas de infecção de B. glabrata por S. mansoni em nove municípios, são mostradas na Figura 1. O segundo trematódeo prevalente em moluscos da microrregião de Belo Horizonte é da família Echinostomatidae cuja larva é a Cercaria macrogranulosa15 (Tabela 3). Em terceiro lugar ocorreu a Cercaria caratinguensis 15 (Tabela 3), família Strigeidae.

Melania tuberculata, o tiarídeo asiático, em expansão em diferentes coleções hídricas do Estado de Minas Gerais, foi encontrado em 7 municípios (Tabelas 1 e 2). Dentre 5.531 exemplares $(25,1 \%)$ nenhum estava infectado com larvas de trematódeos. Do total de moluscos coletados, $378(1,7 \%)$ pertencentes aos gêneros Biomphalaria e Physa estavam infectados com larvas de 8 espécies diferentes de trematódeos incluindo o S. mansoni (Tabela 3).

Dentre 303 exemplares de limneídeos capturados nessa microrregião, nenhum estava infectado com larvas de Fasciola hepatica (Trematoda Fasciolidae).

\section{DISCUSSÃO}

Entre os parasitas transmitidos por planorbídeos no Brasil, o mais investigado por sua importância médica é o Schistosoma mansoni, cujas espécies vetoras naturais são Biomphalaria glabrata, $B$. tenagophila e $B$. straminea e potenciais $B$. peregrina e B. amazonica 12 .

Além de $S$. mansoni outros trematódeos parasitas não humanos completam seus ciclos em planorbídeos e moluscos de gêneros diversos. Larvas de trematódeos paranfistomídeos parasitas de bovinos foram encontradas em $B$. tenagophila, B. peregrina e Drepanotrema kermatoides no Rio Grande do Sul17 18 26. Larvas de Clinostomum heluans, Lutz, 1934, parasita de garças, são encontradas em $B$. glabrata, B. tenagophila e B. straminea. (= Cercaria occelifera 1516 ).

Do total de moluscos de água doce capturados na microrregião de Belo Horizonte 61,3\% pertenciam ao gênero Biomphalaria e 0,7\% estavam infectados com larvas de S. mansoni 
Revista da Sociedade Brasileira de Medicina Tropical 31:449-456, set-out, 1998.

Tabela 1 - Resultado de levantamento malacológico realizado em 13 municípios da microrregião de Belo Horizonte MG, de 1990 a 1996.

\begin{tabular}{|c|c|c|c|c|}
\hline Município & $\begin{array}{c}\text { Coleção hídrica } \\
\text { Pesquisada/positiva }\end{array}$ & Ano & Identificação & Número de exemplares \\
\hline \multirow[t]{13}{*}{ Pedro Leopoldo } & $28 / 5$ & 1990 & Biomphalaria glabrata & 57 \\
\hline & $28 / 9$ & 1990 & Biomphalaria straminea & 506 \\
\hline & $28 / 1$ & 1990 & Biomphalaria tenagophila & 4 \\
\hline & $28 / 3$ & 1990 & Drepanotrema depressissimum & 19 \\
\hline & $28 / 1$ & 1990 & Drepanotrema lucidum & 6 \\
\hline & $28 / 3$ & 1990 & Drepanotrema cimex & 16 \\
\hline & $28 / 4$ & 1990 & Physa cubensis & 17 \\
\hline & $28 / 18$ & 1990 & Physa marmorata & 95 \\
\hline & $28 / 6$ & 1990 & Lymnaea columella & 13 \\
\hline & $28 / 2$ & 1990 & Melania tuberculata & 89 \\
\hline & $28 / 5$ & 1990 & Pomacea sp & 51 \\
\hline & $28 / 1$ & 1990 & Anodontites $s p$ & 1 \\
\hline & $28 / 1$ & 1990 & Idiopyrgus souleyetianus & 2 \\
\hline Subtotal & $28 / 59$ & 1990 & -- & 876 \\
\hline \multirow[t]{11}{*}{ Lagoa Santa } & $26 / 10$ & 1990 & Biomphalaria glabrata & 80 \\
\hline & $26 / 8$ & 1990 & Biomphalaria straminea & 263 \\
\hline & $26 / 1$ & 1990 & Biomphalaria tenagophila & 20 \\
\hline & $26 / 1$ & 1990 & Biomphalaria schrammi & 2 \\
\hline & $26 / 4$ & 1990 & Drepanotrema lucidum & 45 \\
\hline & $26 / 6$ & 1990 & Physa cubensis & 15 \\
\hline & $26 / 12$ & 1990 & Physa marmorata & 114 \\
\hline & $26 / 7$ & 1990 & Lymnaea columella & 35 \\
\hline & $26 / 6$ & 1990 & Melania tuberculata & 140 \\
\hline & $26 / 4$ & 1990 & Pomacea $s p$ & 16 \\
\hline & $26 / 1$ & 1990 & Anodontites sp & 1 \\
\hline Subtotal & $26 / 60$ & 1990 & -- & 731 \\
\hline \multirow[t]{9}{*}{ Santa Luzia } & $26 / 14$ & 1990 & Biomphalaria glabrata & 459 \\
\hline & $26 / 1$ & 1990 & Biomphalaria tenagophila & 1 \\
\hline & $26 / 2$ & 1990 & Drepanotrema lucidum & 14 \\
\hline & $26 / 7$ & 1990 & Drepanotrema cimex & 263 \\
\hline & $26 / 16$ & 1990 & Physa marmorata & 151 \\
\hline & $26 / 1$ & 1990 & Physa cubensis & 2 \\
\hline & $26 / 6$ & 1990 & Lymnaea columella & 41 \\
\hline & $26 / 5$ & 1990 & Pomacea sp & 24 \\
\hline & $26 / 1$ & 1990 & Idiopyrgus souleyetianus & 1 \\
\hline Subtotal & $26 / 53$ & 1990 & -- & 956 \\
\hline \multirow[t]{9}{*}{ Vespasiano } & $53 / 15$ & 1991 & Biomphalaria glabrata & 784 \\
\hline & $53 / 1$ & 1991 & Biomphalaria straminea & 17 \\
\hline & $53 / 13$ & 1991 & Biomphalaria tenagophila & 693 \\
\hline & $53 / 6$ & 1991 & Drepanotrema cimex & 24 \\
\hline & $53 / 28$ & 1991 & Physa marmorata & 363 \\
\hline & $53 / 1$ & 1991 & Physa cubensis & 4 \\
\hline & $53 / 11$ & 1991 & Lymnaea columella & 115 \\
\hline & $53 / 6$ & 1991 & Melania tuberculata & 63 \\
\hline & $53 / 9$ & 1991 & Pomacea sp & 35 \\
\hline Subtotal & $53 / 90$ & 1991 & -- & 2.098 \\
\hline \multirow{6}{*}{ Ribeirão das Neves } & $18 / 16$ & 1990 & Biomphalaria glabrata & 860 \\
\hline & $18 / 2$ & 1990 & Physa cubensis & 15 \\
\hline & $18 / 6$ & 1990 & Physa marmorata & 102 \\
\hline & $18 / 1$ & 1990 & Lymnaea columella & 1 \\
\hline & $18 / 4$ & 1990 & Pomacea sp & 5 \\
\hline & $18 / 1$ & 1990 & Ancylidae & 8 \\
\hline Subtotal & $18 / 30$ & 1990 & -- & 991 \\
\hline Total & $151 / 292$ & $990-195$ & & 5.652 \\
\hline
\end{tabular}


Tabela 2 - Resultado de levantamento malacológico realizado em 13 municípios da microrregião de Belo Horizonte MG, de 1990 a 1996. Município Coleção hídrica

\begin{tabular}{|c|c|c|c|c|}
\hline & Pesquisada/positiva & Ano & Identificação & Número de exemplares \\
\hline \multirow[t]{6}{*}{ Contagem } & $16 / 8$ & 1993 & Biomphalaria glabrata & 494 \\
\hline & $16 / 9$ & 1993 & Biomphalaria tenagophila & 165 \\
\hline & $16 / 2$ & 1996 & Biomphalaria occidentalis & 25 \\
\hline & $16 / 2$ & 1993 & Physa marmorata & 410 \\
\hline & $16 / 1$ & 1996 & Melania tuberculata & 17 \\
\hline & $16 / 1$ & 1996 & Pomacea sp & 3 \\
\hline Subtotal & $16 / 23$ & $1993 / 1996$ & -- & 1.114 \\
\hline \multirow[t]{8}{*}{ Belo Horizonte } & $32 / 20$ & $1990 / 1996$ & Biomphalaria glabrata & 2.135 \\
\hline & $32 / 2$ & 1990/1996 & Biomphalaria straminea & 2.466 \\
\hline & $32 / 8$ & $1990 / 1996$ & Biomphalaria tenagophila & 233 \\
\hline & $32 / 2$ & $1990 / 1996$ & Drepanotrema cimex & 11 \\
\hline & $32 / 18$ & $1990 / 1996$ & Physa marmorata & 454 \\
\hline & $32 / 1$ & 1990/1996 & Lymnaea columella & 6 \\
\hline & $32 / 7$ & 1990/1996 & Melania tuberculata & 5.204 \\
\hline & $32 / 10$ & 1990/1996 & Pomacea sp & 79 \\
\hline Subtotal & $32 / 58$ & $1990 / 1996$ & -- & 10.588 \\
\hline \multirow[t]{6}{*}{ Sabará } & $31 / 31$ & $1991 / 1993$ & Biomphalaria glabrata & 1.173 \\
\hline & $31 / 3$ & 1991/1993 & Biomphalaria straminea & 196 \\
\hline & $31 / 3$ & $1991 / 1993$ & Biomphalaria tenagophila & 100 \\
\hline & $31 / 1$ & 1991/1993 & Biomphalaria peregrina & 38 \\
\hline & $31 / 7$ & $1991 / 1993$ & Physa marmorata & 23 \\
\hline & $31 / 1$ & $1991 / 1993$ & Ancylidae & 1 \\
\hline Subtotal & $31 / 46$ & $1991 / 1993$ & -- & 1.531 \\
\hline \multirow[t]{6}{*}{ Caeté } & $19 / 10$ & 1992 & Biomphalaria glabrata & 200 \\
\hline & $19 / 1$ & 1992 & Biomphalaria straminea & 1 \\
\hline & $19 / 7$ & 1992 & Biomphalaria peregrina & 110 \\
\hline & $19 / 1$ & 1992 & Drepanotrema lucidum & 3 \\
\hline & $19 / 6$ & 1992 & Physa marmorata & 8 \\
\hline & $19 / 1$ & 1992 & Melania tuberculata & 9 \\
\hline Subtotal & $19 / 26$ & 1992 & -- & 331 \\
\hline Raposos & $7 / 7$ & 1992 & Physa marmorata & 237 \\
\hline Subtotal & $7 / 7$ & 1992 & -- & 237 \\
\hline \multirow[t]{5}{*}{ Nova Lima } & $19 / 11$ & 1992 & Biomphalaria glabrata & 673 \\
\hline & $19 / 8$ & 1992 & Biomphalaria straminea & 430 \\
\hline & $19 / 9$ & 1992 & Physa marmorata & 38 \\
\hline & $19 / 1$ & 1992 & Lymnaea columella & 1 \\
\hline & $19 / 1$ & 1992 & Ancylidae & 3 \\
\hline Subtotal & $19 / 30$ & 1992 & -- & 1.145 \\
\hline \multirow[t]{7}{*}{ Betim } & $14 / 8$ & 1993 & Biomphalaria glabrata & 393 \\
\hline & $14 / 4$ & 1993 & Biomphalaria straminea & 139 \\
\hline & $14 / 2$ & 1993 & Biomphalaria tenagophila & 122 \\
\hline & $14 / 6$ & 1993 & Physa marmorata & 12 \\
\hline & $14 / 4$ & 1993 & Lymnaea columella & 97 \\
\hline & $14 / 1$ & 1993 & Melania tuberculata & 9 \\
\hline & $14 / 2$ & 1993 & Pomacea sp & 3 \\
\hline Subtotal & $14 / 27$ & 1993 & -- & 775 \\
\hline \multirow[t]{3}{*}{ Rio Acima } & $19 / 10$ & 1992 & Biomphalaria glabrata & 612 \\
\hline & $19 / 1$ & 1992 & Biomphalaria straminea & 75 \\
\hline & $19 / 7$ & 1992 & Physa marmorata & 6 \\
\hline Subtotal & $19 / 18$ & 1992 & -- & 693 \\
\hline Total & $157 / 235$ & 1990/1996 & -- & 16.414 \\
\hline
\end{tabular}




\begin{tabular}{|c|c|c|c|c|c|}
\hline \multirow{2}{*}{$\begin{array}{c}\text { Molusco } \\
\text { Gênero/espécie }\end{array}$} & \multirow{2}{*}{$\begin{array}{c}\text { Total } \\
\text { coletado }\end{array}$} & \multirow{2}{*}{$\begin{array}{l}\text { Número de } \\
\text { infectado }\end{array}$} & \multirow{2}{*}{$\begin{array}{l}\text { Taxa de } \\
\text { infecção }\end{array}$} & \multicolumn{2}{|c|}{ Trematódeo } \\
\hline & & & & larva & família \\
\hline \multirow[t]{5}{*}{ B. glabrata } & 7.920 & 156 & 1,9 & S. mansoni & Schistosomatidae \\
\hline & & 48 & 0,6 & C. caratinguensis & Strigeidae \\
\hline & & 94 & 1,2 & C. macrogranulosa & Echinostomatidae \\
\hline & & 9 & 0,1 & C. minense & -- \\
\hline & & 3 & $-0,1$ & C. hemiura & Derogenidae \\
\hline \multirow[t]{5}{*}{ B. straminea } & 4.093 & 24 & 0,6 & C. caratinguensis & Strigeidae \\
\hline & & 10 & 0,2 & C. macrogranulosa & Echinostomatidae \\
\hline & & 1 & $-0,1$ & C. occelifera & Clinostomatidae \\
\hline & & 1 & $-0,1$ & C. não identificada & -- \\
\hline & & 1 & $-0,1$ & C. não identificada & -- \\
\hline B. tenagophila & 1.338 & 2 & 0,1 & C. caratinguensis & Strigeidae \\
\hline P. marmorata & 1.776 & 29 & 1,6 & C. macrogranulosa & Echinostomatidae \\
\hline Total & 15 & 378 & 2,5 & -- & -- \\
\hline
\end{tabular}

(B. glabrata) com focos em 9 municípios (Figura 1) e 1,0\% com larvas de outros trematódeos. Biomphalaria glabrata, B. straminea e $B$. tenagophila, apresentaram infecção com larvas de trematódeos das seguintes famílias: Schistosomatidae (S. mansoni), Strigeidae, Echinostomatidae, Clinostomatidae, Derogenidae e Cercaria minense 15 além de dois tipos de larvas não identificadas (Tabela 3). As larvas de trematódeos que ocorreram com maior freqüência nessa microrregião foram: S. mansoni, $0,7 \%$, (Biomphalaria); Echinostomatidae, 0,6\% (Biomphalaria e Physa) e Strigeidae, 0,3\% (Biomphalaria). Do gênero Physa, 1,6\% dos moluscos coletados estavam infectados com larvas de Echinostomatidae (Tabela 3 ). Biomphalaria glabrata apresentou o índice mais elevado de infecção natural, com larvas de 5 trematódeos, nos 310 exemplares parasitados $(1,4 \%)$, mostrando suscetibilidade maior não somente ao $S$. mansoni, mas a vários parasitas.

A redução do número de focos de transmissão da esquistossomose no município de Belo Horizonte foi muito acentuada em relação ao levantamento anterior22. Só foram coletados moluscos infectados no Barreiro de Baixo20 (foco extinto durante este estudo) e em cinco pontos do córrego da Mangueira na região do Gorduras.

Na microrregião de Belo Horizonte, a taxa de prevalência de esquistossomose em escolares, nas últimas pesquisas, estava em torno de $8,3 \% 38$.
Cercárias de Fasciola hepática não foram encontradas em nenhum dos limneídeos coletados nessa região.

Esses resultados vem demonstrar a necessidade de mais pesquisas sobre os ciclos biológicos destes trematódeos, parasitas não humanos, pois, os estudos sobre sistemática desses helmintos, em geral, identificam somente os vermes adultos e os hospedeiros definitivos vertebrados 1125 , enquanto as formas larvárias e os hospedeiros intermediários invertebrados são pouco conhecidos. Ruiz15 16 descreveu diferentes formas larvárias de trematódeos em moluscos de Minas Gerais mas os vermes adultos e os hospedeiros vertebrados ainda são pouco conhecidos. O conhecimento das áreas geográficas de ocorrência dos moluscos hospedeiros intermediários de trematódeos parasitas de animais de interesse econômico é importante para se detectar os riscos de transmissão de parasitoses aos rebanhos nessas regiões.

Portanto, apesar das dificuldades encontradas em pesquisas para se fechar experimentalmente os ciclos biológicos de trematódeos parasitas não humanos, é necessário melhorar o conhecimento sobre esses parasitas bastante comuns, principalmente na região Sudeste.

\section{AGRADECIMENTOS}

Aos técnicos Moacyr Rodrigues da Silva e José Geraldo Amorim da Silva pelo trabalho de campo. 


\section{REFERÊNCIAS BIBLIOGRÁFICAS}

1. Andrade, RM. Ecologia do Autralorbis glabratus (Pulmonata: Planorbidae) em Belo Horizonte, MG, Brasil. IV Infecção simples e mistas dos caramujos por trematódeos. Revista Brasileira de Malariologia e Doenças Tropicais 17:371-377, 1956a.

2. Andrade, RM. Ecologia do Autralorbis glabratus em Belo Horizonte, Brasil. V Infecção natural por "Cercaria macrogranulosa" Revista Brasileira de Biologia, p. 305-314, 1956b.

3. Araújo MC, Del Rio BR, Mello GG, Marques RN. Levantamento Parasitológico da esquistossomose mansoni em escolares da rede municipal de Belo Horizonte. Revista da Sociedade Brasileira de Medicina Tropical 19 (supl):63-64, 1986.

4. Deslandes N. Técnica de dissecação e exame de planorbídeos. Revista do Serviços Especiais de Saúde Pública 4:371-382, 1951.

5. Freitas JR, Santos MBL, Lima LZ, Rocha LA, Souza CP. Distribuição dos moluscos hospedeiros intermediários do Schistosoma mansoni na Bacia da Pampulha, Belo Horizonte, MG - atualização de dados. Ciência e Cultura 43 (supl):641-642, 1991.

6. Guimarães CT, Antunes-de-Souza M, Soares DM, Souza CP. Levantamento malacológico em parques urbanos de Belo Horizonte, Minas Gerais - Brasil. Cadernos de Saúde Pública 13:313-316, 1997.

7. Guimarães CT, Souza CP, Carvalho OS, Katz N. Sobre um foco urbano de esquistossomose em área metropolitana da região sudeste do Brasil.Revista de Saúde Pública de São Paulo 27:210-213, 1993.

8. Lambertucci JR, Rocha RS, Carvalho OS, Katz N. Esquistossomose mansoni em Minas Gerais. Revista da Sociedade Brasileira de Medicina Tropical 20:4752, 1987.

9. Martins AV, Martins G, Falcão AL. Distribuição geográfica dos planorbídeos e seus índices de infecção pelas cercárias de Schistosoma mansoni no município de Belo Horizonte, MG. X Congresso Brasileiro de Higiene, Curitiba, 1953.

10. Martins AV, Versiani W. Schistosomose mansoni em Belo Horizonte. Brasil Médico 52:481-482, 1938.

11. Miranda MA. Paramphistomidae Fischoeder, 1901 (Trematoda) parasitos de ruminantes domésticos em alguns estados das regiões Norte e Nordeste do Brasil. Tese de Doutorado, Universidade Federal de Minas Gerais, Belo Horizonte, 1997.

12. Paraense WL. Planorbídeos e limneídeos brasileiros vetores de parasitos. In: Resumos do XV Encontro Brasileiro de Malacologia, Santa Catarina, p. 32, 1997.
13. Paraense WL, Deslandes N. Observations on the morphology of Australorbis glabratus. Memórias do Instituto Oswaldo Cruz 53:87-103, 1955.

14. Paulini E, Dias EP, Fiuza H. Contribuição a epidemiologia da esquistossomose em Belo Horizonte. Revista Brasileira de Malariologia e Doenças Tropicais 19:571-606, 1967.

15. Ruiz JM.Contribuição ao estudo das formas larvárias de trematoides brasileiros. Fauna de Belo Horizonte e Jaboticatubas, Estado de Minas Gerais. Memórias do Instituto Butantam 24:45-62, 1952.

16. Ruiz JM.Contribuição ao estudo das formas larvárias de trematoides brasileiros. Memórias do Instituto Butantam 25:77-89, 1953.

17. Santos ICS, Laranja RJ, Martins JR, Ceresér VH. Hospedeiros intermediário de Paramphistomum (Fischoeder, 1901) Biomphalaria tenagophila (Orbigny) em Guaiba, Rio Grande do Sul. Nota prévia. In: Anais do $18^{\circ}$ Congresso Brasileiro de Medicina Veterinária, Balneario de Camboriu, Santa Catarina, p.120, 1982.

18. Santos ICS, Martins JRS, Laranja RJ. Paranfistomose no Rio Grande do Sul.A Hora Veterinária, Ed. Extra, no I, p. 48-56, 1995.

19. Silva RE, Melo AL, Pereira LH, Frederico LF. Levantamento malacológico da bacia hidrográfica do lago Soledade, Ouro Branco (Minas Gerais, Brasil). Revista do Instituto de Medicina Tropical de São Paulo 36:437-444, 1994.

20. Souza CP, Araujo N, Jannotti-Passos LK, Guimarães CT. Production of Schistosoma mansoni cercariae by Biomphalaria glabrata from a focus in Belo Horizonte, Minas Gerais. Revista do Instituto de Medicina Tropical de São Paulo 36:485-489, 1994.

21. Souza CP, Mendes NM. Repovoamento de criadouros de Biomphalaria glabrata após tratamento com niclosamida. Revista do Instituto de Medicina Tropical de São Paulo 33:297-302, 1991.

22. Souza CP, Pereira JP, Rodrigues MS. Atual distribuição geográfica dos moluscos hospedeiros intermediários do Schistosoma mansoni em Belo Horizonte, MG, Brasil.Memórias do Instituto Oswaldo Cruz 76:383-391, 1981.

23. Souza CP, Ribeiro PRB, Guimarães CT, JannottiPassos LK, Souza MA. Esquistossomose: nova ocorrência de Biomphalaria straminea em Belo Horizonte, Minas Gerais. Cadernos de Saúde Pública 12:541-544,1996. 
Revista da Sociedade Brasileira de M edicina Tropical 31:449-456, set-out, 1998.

24. Teixeira MJ. A schistosomose mansônica na infância em Belo Horizonte. Tese de concurso. Faculdade de Medicina, Imprensa Oficial de Belo Horizonte, 1919.

25. Travassos L, Freitas JFT, Kohn A. Trematódeos do Brasil.Memórias do Instituto Oswaldo Cruz, Tomo 67, fascículo único, 1969.
26. Veitenheimer-Mendes JL, Almeida-Caon JEM. Drepanotrema kermatoides (Orbigny, 1835) (Mollusca: Planorbidae), hospedeiro de um paranfistomídeo (Trematoda) no Rio Grande do Sul, Brasil.Memórias do Instituto Oswaldo Cruz 84:107-111, 1989. 